\title{
Domande alla storia. Presentazione
}

Storicamente, 2 (2006).

ISSN: 1825-411X. DOI: 10.12977/stor537

\section{Presentazione}

Mai come oggi la storia desta l'interesse dell'opinione pubblica; eppure mai come oggi le questioni che animano il dibattito storiografico sono fatte oggetto di distorsioni nell'impostazione e nei contenuti a causa di una separazione crescente tra la comunicazione scientifica dei centri di ricerca e la divulgazione storica dei mezzi di comunicazione di massa.

Mentre si discuteva di fine della storia, la storia si rimetteva in moto a partire da eventi traumatici, dall'11 Settembre alla seconda guerra nel Golfo, dalla ripresa dell'Intifada agli attentati di Madrid e Londra. Un'analisi attenta di questi avvenimenti ne individua le radici in questioni e problemi mai risolti, lontani nel tempo, che solo la lente dello storico può aiutare a mettere a fuoco.

Con gli eventi si sono riaccese anche discussioni intorno a concetti fondamentali. La questione dell'identità, la globalizzazione e la rimodellizzazione di un mondo ormai irriducibile alle semplici categorie di Oriente e Occidente, il problema delle religioni e del loro rapporto con il potere e le istituzioni, il tema della democrazia e della sua 'esportabilità' sono solo alcuni dei punti toccati dal dibattito culturale più aggiornato.

Ci sembra quindi opportuno ripartire dal duplice insegnamento di Marc Bloch - - «se non ci si china sul presente è impossibile capire il passato» e analogamente " l'incomprensione del presente nasce fatalmente dallignoranza del passato» - e interrogare la storia secondo questa prospettiva, con la consapevolezza che l'indagine scientifica su ciò che è 
stato aiuta a capire ciò che è attuale e viceversa.

L'iniziativa Domande alla storia, promossa dal Dipartimento di Politica, Istituzioni, Storia e dal Dipartimento di Discipline Storiche dell'Università degli Studi di Bologna assieme a UniboCultura, si propone dunque di colmare la distanza che separa la storia degli storici e la storia troppo spesso travisata dall'uso mediatico che ne viene fatto, avvicinando il pubblico ad alcuni dei temi storiografici al centro del dibattito attuale attraverso la mediazione di storici di fama internazionale.

II ciclo di conferenze, inoltre, rinsalderà il legame che unisce l'Alma Mater alla sua città, Bologna, facendo uscire i risultati della ricerca dalle mura degli istituti universitari.

Programma 2006-2007

Ciclo di conferenze

Ore 17,30

Sala dei Poeti, Facoltà di Scienze Politiche Strada Maggiore 45, Bologna

\section{6 febbraio}

Tiziano Bonazzi (Università degli Studi di Bologna)

Gli americani sono Europei?

\section{0 marzo}

Roberto Chiarini (Università degli Studi di Milano)

Resistenza larga o resistenza stretta?

[[youtube]]ymXoXpvDjt8[[youtube]]

\section{3 aprile}

Prof. Ernesto Galli della Loggia (Università degli Studi di Perugia)

Esiste l'identità repubblicana? 
Conferenza

[[youtube]]18i03p1pO5Y[[youtube]]

Dibattito

[[youtube]]QD1iiOB3oHU[[youtube]]

\section{8 maggio}

Victoria de Grazia (Columbia University, New York- Istituto Universitario Europeo, Fiesole)

La società dei consumi è democratica?

Conferenza

[[youtube]]T0O6uYka-s8[[youtube]]

Dibattito

[[youtube]]GH6o8eZ-Q8c[[youtube]]

\section{5 giugno}

Aldo Schiavone (Università degli Studi di Firenze)

Il diritto: un'invenzione dell'Occidente?

Conferenza

[[youtube]]Dt8rwHwMCDE[[youtube]]

Dibattito

[[youtube]]ssI7gB7plro[[youtube]]

\section{1 settembre}

Marcello Flores (Unive rsità degli Studi di Siena)

Il Novecento: il secolo della violenza?

[[youtube]]o9FL8YWnTZg[[youtube]]

\section{9 ottobre}

Franco Cardini (Università degli Studi di Firenze)

Esiste un'identità europea? 
Conferenza

[[youtube]]FwHrM0FRk5Q[[youtube]]

Dibattito

[[youtube]]QD1iiOB3oHU[[youtube]]

\section{6 novembre}

Emilio Gentile (Università degli Studi di Roma La Sapienza)

Cos'è il fascismo?

Conferenza

[[youtube]]FFluucXGUKY[[youtube]]

Dibattito

[[youtube]]WyJp0QkH9DU[[youtube]]

\section{4 dicembre}

Carlo Ginzburg (UCLA, Los Angeles)

Terrore: quale terrore?

Le riprese delle conferenze di Ernesto Galli della Loggia, Victoria De Grazia e Aldo Schiavone sono state effettuate da Manuela Colin del CITAM 